\title{
A Multi-Sensory Approach Integrating User Preferences
}

\author{
Lahcene Aid, Lynda Zaoui, and Sid Ahmed Mostefaoui
}

\begin{abstract}
The creation and maintenance of a comfortable internal environment is one of the major concerns of the smart home designers. Indoor comfort depends on the individual's physiology and psychology. It is essential to have a full understanding of occupants' satisfaction in order to maintain a comfortable indoor environment. However, it is important to develop a model that adapt to the user's desires and needs. This paper presents a novel multi-sensory approach based on the DEVS (Discrete Event System Specification) and the theory of fuzzy logic for modeling and simulation of occupant's preferences. The approach takes into consideration the interrelations between all senses namely the thermal, visual, acoustical and olfactory sensory systems on the sensation of global comfort.
\end{abstract}

Index Terms-Comfort, devs, fuzzy logic, modeling, smart home.

\section{INTRODUCTION}

Most of the works examining the issue of comfort of building's occupants in indoor environments were focused on thermal comfort [1]-[4]. Ensuring, or even maximizing occupants' thermal comfort alone is not sufficient for their overall satisfaction. Moreover, the perception of indoor environment quality is influenced by many aspects such as air quality, ventilation, lighting, and noise.

Occupants in buildings are exposed simultaneously to all indoor environmental parameters (thermal, visual, acoustic environment and air quality) and their evaluation of the indoor environment is influenced by the combined effect of different environmental parameters. On the one hand, several studies have indicated that people tend to reject automatic systems when the control algorithm does not respect their personal preferences [5].On the other hand, incorporating and integrating user preferences in an automatic control algorithm is a complex task.

Users are not able to specify what values are acceptable to them in terms of absolute numbers. Therefore, user preferences have to be captured indirectly. The main goal of this paper is to propose a model using the DEVS formalism integrated with the theory of fuzzy logic to describe and simulate a complex system of user's preferences.

One of the few works done in this area is a primitive approach proposed by Vainio et al. [6], where a fuzzy controller adjusts the weights of a few predefined factors that determine whether performing a specific action is in accordance with the user's preferences or not, e.g. turning on the lamp if the light level is less than a certain amount.

Manuscript received September 30, 2012; revised December 21, 2012.

The authors are with the System-Signal and Data laboratory LSSD, University of Science and Technology Mohamed Boudiaf USTO, Oran, Algeria (e-mail: a_lahcen@esi.dz).
However, they do not take into account that the user's evaluation of the indoor environment is influenced by the combined effect of different environmental parameters.

This paper is organized as follows: The next section gives an overview of the basic concepts of DEVS and the theory of fuzzy logic. In Section III, we present the Smart Home DOMUS. In Section IV, we describe our model of the user's preferences. The last section concludes our work.

\section{BACKGROUND}

In this section we introduce the formalism DEVS and some essential concepts, terminology, and arithmetic of fuzzy sets and fuzzy logic.

\section{A. The Devs Formalism}

BP Zeigler defined in [7], a formal specification of discrete event models. This formalism was introduced as an abstract universal formalism independent of the implementation. The DEVS formalism is based on the definition of two types of models: atomic models and coupled models. The atomic models used to represent the basic behavior of the system. Coupled models are defined by a set of sub atomic models and / or coupled models to represent the internal structure of the system through coupling between models.

The atomic model provides an autonomous description of system behavior, defined by states and input/output functions and internal transitions of the model. The evolution of the model is done by state change according to external stimuli (via an input) or internal (via a transition function). These state changes are intended to determine the behavioral response of the system to these stimuli.

Formally, an atomic model $M$ is specified by a 7-uplet:

$$
A M=\left(X, Y, S, \delta_{i n t}, \delta_{e x t}, \lambda, t a\right)
$$

where

$X$ : is the set of external events (input).

$Y$ : is the set of output events.

$S:$ is the set of states.

$\delta_{\text {int }}$ int $: S \rightarrow S$ is the internal transition function caused by the occurrence of internal events.

$\delta_{\text {ext }}$ ext: $Q \times X \rightarrow S$ is the external transition function caused by the occurrence of external events where

$Q=\{(s, e) \mid s \in S, O \leq e \leq t a(s)\}$ : total states and e describes the elapsed time since the system made a transition to the current state s.

$\lambda: S \rightarrow Y$ is the output function

$t a$ : time-advance, the function of lifetime of state, represents the maximum time during which the model remains in a state.

Fig. 1 shows the description of a DEVS atomic model.

To describe a more complex system we interconnect 
several atomic models to form a coupled model. This new model can be used as a base model in a higher level description is the hierarchical aspect of the formalism [8].

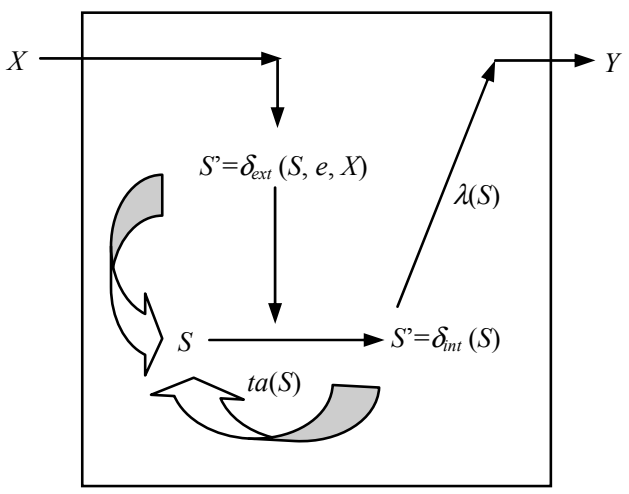

Fig. 1. Description of a DEVS atomic model

A coupled DEVS model named CM is a structure:

$$
<X, Y, D,\left\{M_{d} / d \in D\right\}, E I C, E O C, I C>
$$

where

$X$ : set of possible inputs of the coupled model.

$Y$ : set of possible outputs of the coupled model.

$D$ : set of names associated to the model components.

$M_{d}$ : set of the coupled model components, these components are either atomic or coupled DEVS model.

$E I C$ : set of External Input Coupling.

EOC: set of External Output Coupling.

$I C$ : set of internal couplings.

Fig. 2 shows the description of a couple DEVS model.

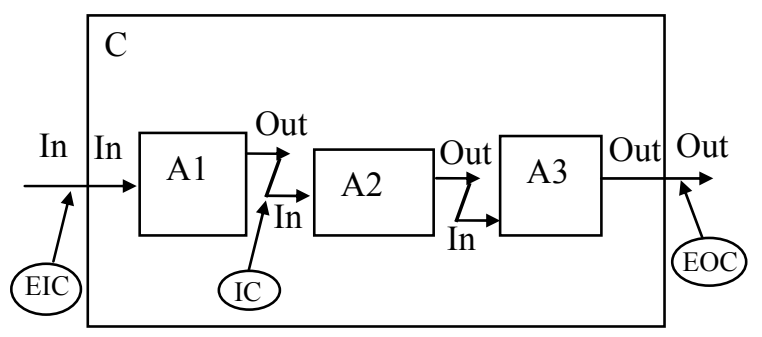

Fig. 2. Description of a coupled DEVS

\section{B. Fuzzy Logic}

Fuzzy systems theory is based on uncertainty and on imprecision. Uncertainty is very natural to humans and people usually make decisions based on indiscrete observations [9].

Linguistic variables are used in fuzzy systems as a method for performing calculations. The values of linguistic variables can be presented using membership functions (MFs). The MF is a curve that defines how each point in the input space is mapped to a membership value (or degree of membership) between 0 and 1. Many different types of MF curves are available for these applications such as triangular, trapezoidal, Gaussian distribution curve etc.

Fuzzy inference systems (FIS) are one of the most famous applications of fuzzy logic and fuzzy sets theory [10].

The realization of an inference system consists of three steps: fuzzification, fuzzy rule inference and defuzzification.

Fuzzification consists of transforming a real measured value into fuzzy values of linguistic variables. These fuzzy values receive a degree of membership based on the real value and the respective membership function.

The second step is called fuzzy rule inference whereby some sets of fuzzy logic operators and production rules are defined. The most common rule is called IF-THEN rule which can be used to formulate the conditional statements that comprise fuzzy logic.

Last, the defuzzification process is to convert the output of the fuzzy rules into a scalar, non-fuzzy value.

\section{DESCRIPTION OF THE DOMUS SMART HOME}

As part of the Carnot institute LSI, the MultiCom research group [11] built a prototype of a smart apartment $\left(40^{2}\right)$. This apartment consists of a set of pieces embodying a classic apartment (office, bedroom, bathroom and kitchen with dining room) and has a real furniture; A pictures of the DOMUS smart home is shown in fig. 3. The entire apartment is controlled by a home automation system. This home automation system also allows interaction with tangible objects and collection activity traces.
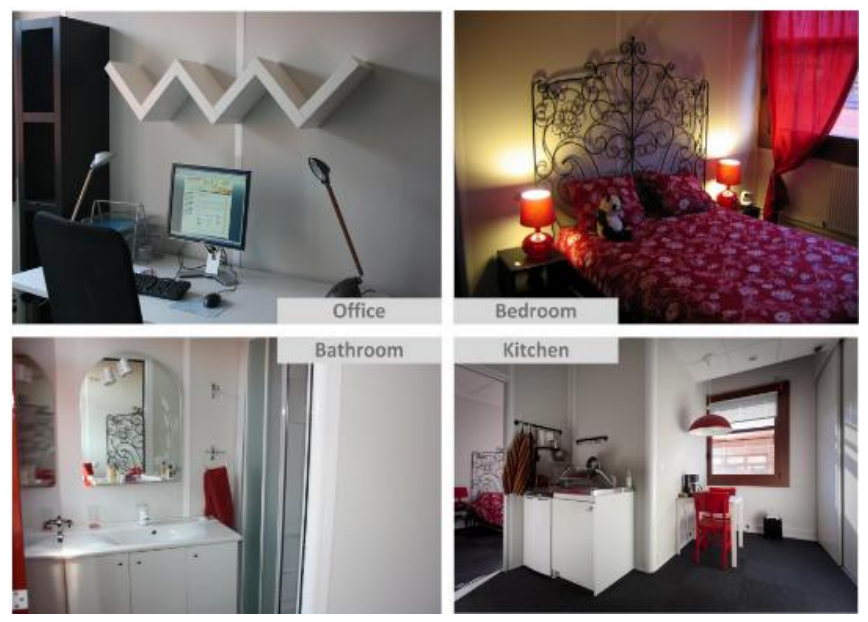

Fig. 3. The DOMUS smart home

\section{PREFERENCE Model CONSTRUCTION}

Modeling and simulation with DEVS has become a reference to the question of coupling of heterogeneous models. The classic DEVS formalism does not take into account inaccuracy and uncertainty on the events and the states. On the other hand, the multi-formalism DEVS enables the integration of many other formalisms or modeling methods. However, the integration of fuzzy logic is the best choice, as humans perceive their environment with fuzzy variables.

\section{A. Experimental Data Set}

Experimental data set is the most significant part of a research. To develop our model, we have used the Multicom Domus Dataset [12]; a total of 20 people (8 males, 12 females) were asked to participate in the research work. They were asked to spend about 1 hour and a half in the intelligent flat. The experiment was divided in 3 slots of 20 to 30 minutes, each one of them in a specific room with a specific activity. Also, inhabitants were asked to fill a form every five minutes in order to understand their perception of comfort with a 
sensorial semantic (see Table I) and a technical semantic (see Table II). Each of these variables was presented in the form of a Likert scale to the inhabitant. Answers were transposed into quantitative data for analysis, ranging from 0 for « very unpleasant » to 10 for « very pleasant ».

The questionnaire was implemented in an electronic and mobile way (fig. 4) in order to facilitate the user's annotations [13].

TABLE I: USER's PERCEPTIONS QUESTIONNAIRE

\begin{tabular}{ll}
\hline \hline Name & (Likert) Scale legend \\
\hline Global comfort & Very unpleasant to very pleasant \\
Thermal comfort & Very unpleasant to very pleasant \\
Lighting comfort & Very unpleasant to very pleasant \\
Air quality & Very unpleasant to very pleasant \\
Acoustic comfort & Very unpleasant to very pleasant \\
\hline \hline
\end{tabular}

TABLE II: USER's FEELINGS QUESTIONNAIRE

\begin{tabular}{ll}
\hline \hline Name & (Likert) Scale legend \\
\hline Temperature & Very cold to very hot \\
Humidity & Very humid to very dry \\
Luminosity & Very dark to very bright \\
Ventilation / air speed & Very slow to very high \\
Smell & Very unpleasant to very pleasant \\
Noise level & Very low to very high \\
Agreeableness of & Very unpleasant to very pleasant \\
background noise & \\
\hline \hline
\end{tabular}

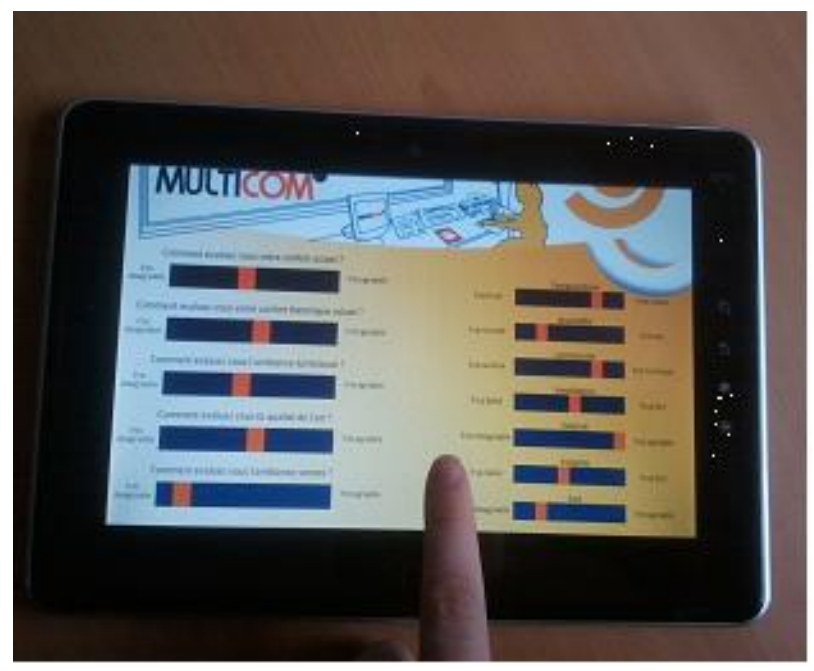

Fig. 4. Interface of questionnaire

\section{B. Structure of the User's Preferences with DEVS}

To specify the preference system we have divided it on five sub-systems as shown in the fig.5, where:

$X_{T}$ is the set inputs of the thermal preferences model $=$ \{External Temperature, Internal Temperature, Air speed, Humidity, Window position, Ventilation speed, Lamp, Venetian blinds position, Thermostat, Time, weekday

$X_{V}$ is the set inputs of the visual preferences model = \{Internal Luminosity, External Luminosity, Venetian blinds position, Lamp, Time, weekday $\}$.

$X_{A}$ is the set inputs of the acoustic preferences model $=$ \{Noise Level, Ventilation speed, Window position, Door position, Time, weekday\}.

$X_{O}$ is the set inputs of the olfactory preferences model $=$
\{Air speed, Ventilation, Door position, C02- Concentration, Window position, Time, weekday .

\section{Formal Specification of the Preferences}

The user's preferences model is a coupled DEVS model composed of five atomic models namely Thermal preferences model, visual preferences model, Acoustic preferences model, olfactory preferences model and Global comfort model.

Preferences $=<X, Y, D,\left\{M_{d} \mid d \in D\right\}, E I C, E O C, I C$, SELECT>

where

$X=\left\{(p, v) / p \in\right.$ IPorts, $\left.v \in X_{p}\right\}$ is the set of inputs.

$Y=\left\{(p, v) / p \in\right.$ OPorts, $\left.v \in Y_{p}\right\}$ is the set of output.

$D$ set of names associated to the model components.

$D=\{$ Thermal preferences, Visual preferences, Acoustic preferences, Olfactory preferences, Global comfort $\}$.

$M_{d}$ : set of the coupled model components.

$M_{\text {Thermal preferences }}$ is the sub-system of the thermal user's preferences.

$M_{\text {Visual preferences }}$ is the sub-system of the Visual user's preferences.

$M_{\text {Acoustic preferences }}$ is the sub-system of the Acoustic user's preferences.

$M_{\text {Olfactory preferences }}$ is the sub-system of the olfactory user's preferences.

$M_{\text {Global comfort }}$ is the sub-system of the user's global comfort. $\mathrm{EIC}=\{(($ Preference, input $),($ Thermal preferences, input $))$, ((Preference, input), (Visual preferences, input)), ((Preference, input), (Acoustic preferences, input)), ((Preference, input), (Olfactory preferences, input)), ((Preference, input), (Global comfort, input))\}.

$\mathrm{EOC}=\{(($ Global comfort, output $),($ Preference, output $))\}$.

$\mathrm{IC}=\{(($ Thermal preferences, output), (Global comfort, input)), ((Visual preferences, output), (Global comfort, input)), ((Acoustic preferences, output), (Global comfort, input)), ((Olfactory preferences, output), (Global comfort, input)) $\}$.

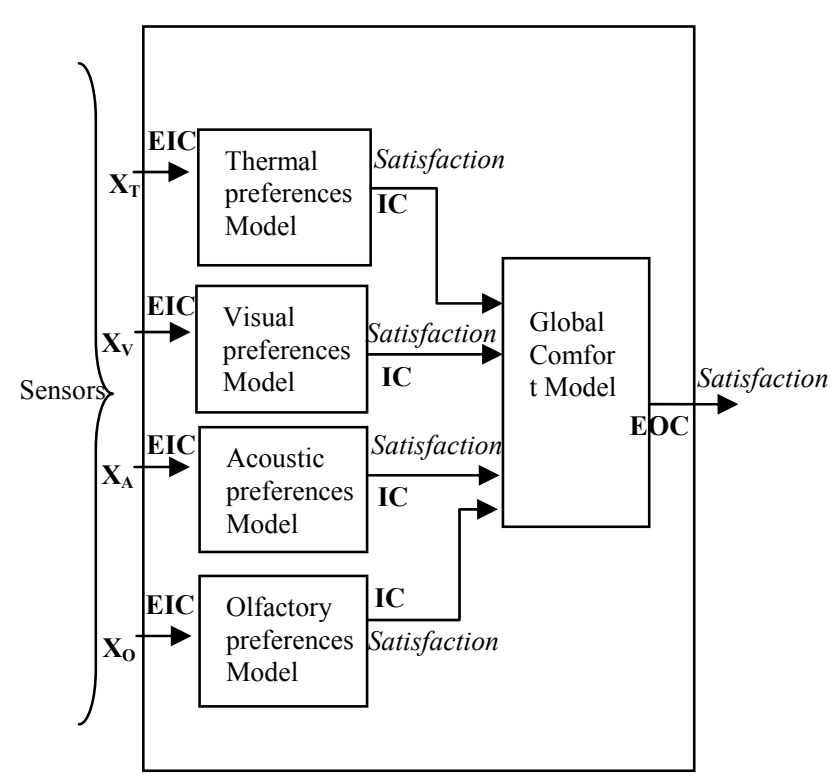

Fig. 5. Structure of the user's preferences coupled DEVS model. 


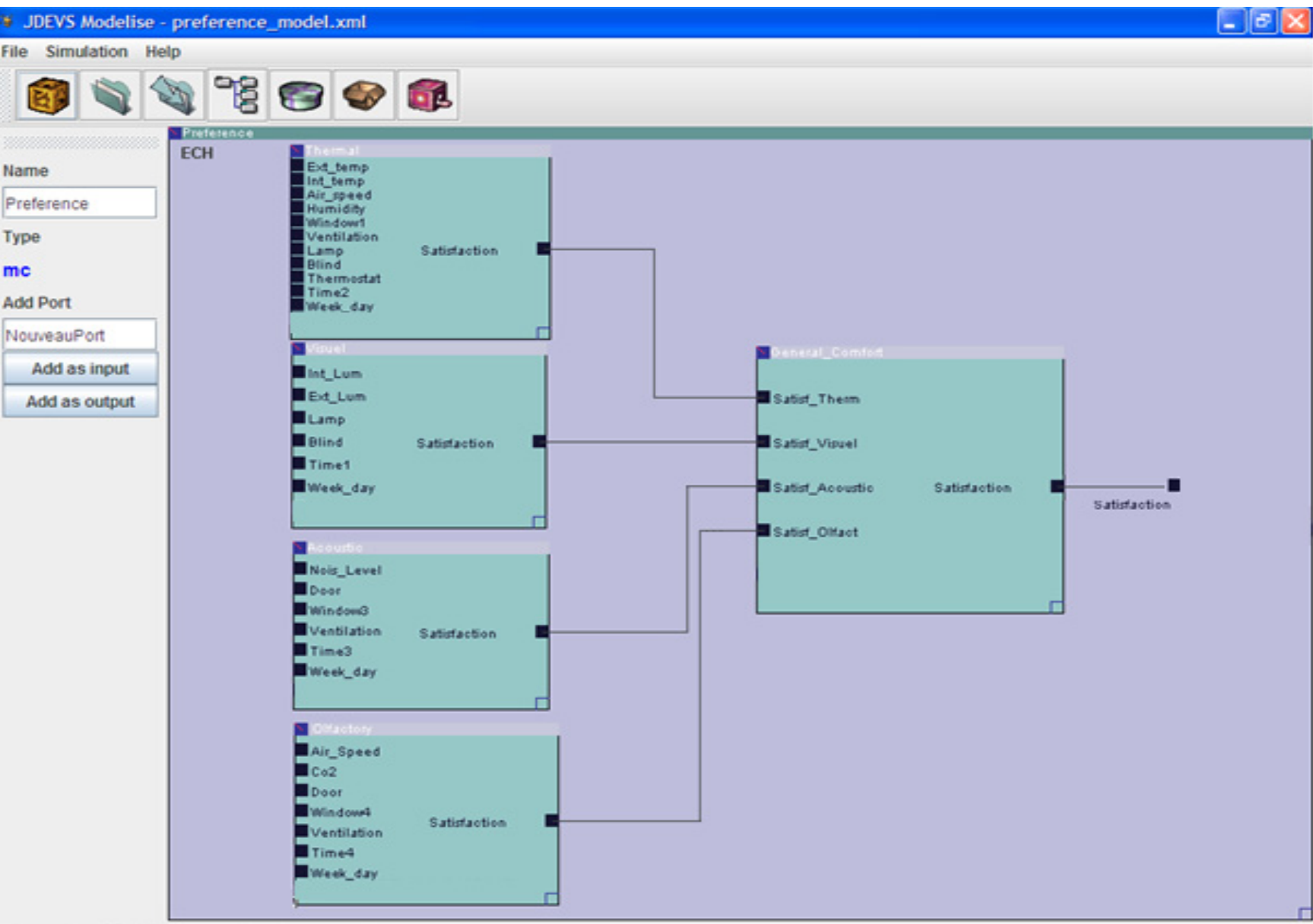

Fig. 6. Representation of the model under JDEVS.

The Table III summarizes the linguistic variables used in the Fuzzification for the sensory inputs.

We have added two fuzzy inputs variables weekday and Time. The Weekday consists of seven non overlapping values, named by the weekdays. The linguistic output variable for each system is Satisfaction with five values: 'very unpleasant', 'unpleasant', 'neutral', 'pleasant' and 'very pleasant'.

We have used the standard Mamdani fuzzy inference system [9]. Also, we have used the Multicom Domus Dataset to define the fuzzy rules and the fuzzy outputs are defuzzified by the center of gravity procedure.

\section{A. Thermal Preferences Model}

The formal specification of the Thermal preferences in DEVS is as follows:

Thermal preferences $=\left(X, Y, S, \delta_{\text {int }}, \delta_{\text {ext }}, \lambda, t a\right)$

where:

$X=\{$ External Temperature, Internal Temperature, Air speed, Humidity, Window position, Ventilation speed, Lamp, Venetian blinds position, Thermostat, Time, weekday .

$Y=\{$ very unpleasant, unpleasant, neutral, pleasant, very pleasant $\}$

$S$ : Fuzzified values.

$\delta_{\text {int }}=\varnothing$.

$\delta_{\text {ext }}=$ FIS _thermal(X) fuzzy inference system, it receives input $(X)$.

$t a=\infty$.
We have used the same specification for the other sub-systems (Visual preferences, Acoustic preferences, olfactory preferences, Global comfort) with some changes at the external transition function $\left(\delta_{e x t}\right)$ and the inputs $(X)$.

\section{B. Representation of the Proposed Model Using the Modeling Tool JDEVS}

Simulation tools have become essential. They allow to study and understand complexes actions that may be impossible to study in situ. Currently there are several modeling and simulation environments based on the DEVS formalism allowing the representation of different atomic or coupled DEVS models. We chose the environment JDEVS [14] as a tool for modeling and simulation for our proposal model.

JDEVS provides a different approach than the existing tools. In terms of flexibility and genericity of use, it can provide the high-level approach of a general formalism. In terms of features, abstraction, components and interfaces, JDEVS provides the advantages of a domain specific modelling environment. With JDEVS, it is also possible to specify, store, retrieve, couple and simulate different kinds of models without having to specify how those models should be simulated.

The Fig. 6 shows the proposed model of user's preferences modeled on the environment JDEVS. This model consists of several atomic models.

The simulation shows the importance of a multi-sensory approach to ensure the comfort in the indoor environment and improve the level of the occupant's satisfaction. 
TABLE III: THE LINGUISTIC VARIABLES CORRESPONDING TO THE SENSORY DATA

\begin{tabular}{|c|c|c|}
\hline FIS & Linguistic Variable & Values \\
\hline \multirow{9}{*}{ 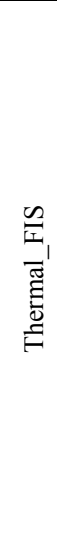 } & External Temperature & \multirow{2}{*}{$\begin{array}{l}\text { Cold, Cool, Slightly Cool, } \\
\text { Neutral, Slightly Warm, } \\
\text { Warm, Hot }\end{array}$} \\
\hline & Internal Temperature & \\
\hline & Air speed & Low, Medium, High \\
\hline & Relative Humidity & Low, Medium, High \\
\hline & Window position & Open, Closed \\
\hline & Ventilation speed & Low, Medium, High \\
\hline & Lamp & Off, On \\
\hline & $\begin{array}{l}\text { Venetian } \\
\text { blinds position }\end{array}$ & Down, Medium, Up \\
\hline & Thermostat & Low, Medium, High \\
\hline \multirow{4}{*}{ 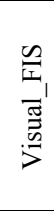 } & Internal Luminosity & \multirow{2}{*}{$\begin{array}{l}\text { Very dark, Dark, Medium } \\
\text { Bright, Bright } \\
\text { Very Bright }\end{array}$} \\
\hline & External Luminosity & \\
\hline & $\begin{array}{l}\text { Venetian } \\
\text { blinds position }\end{array}$ & Down, Middle, Up \\
\hline & Lamp & Off, On \\
\hline \multirow{4}{*}{ 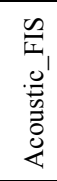 } & Noise Level & $\begin{array}{l}\text { Very Low, Low, Medium, } \\
\text { High, Very High }\end{array}$ \\
\hline & Window position & Open, Closed \\
\hline & Ventilation speed & Low, Medium, High \\
\hline & Door position & Open, Closed \\
\hline \multirow{5}{*}{ 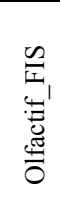 } & Air speed & Slow, Medium, High \\
\hline & Ventilation speed & Low, Medium, High \\
\hline & Door position & Open, Closed \\
\hline & C02-Concentration & $\begin{array}{l}\text { Very Low, Low, Medium, } \\
\text { High, Very High }\end{array}$ \\
\hline & Window position & Open, Closed \\
\hline
\end{tabular}

\section{CONCLUSION AND FUTURE WORK}

In this paper, we presented the modeling and simulation of user preferences. We developed an original multi-sensory model able to ensure the satisfaction of occupants. Also, we combined the DEVS formalism and the theory of fuzzy logic to cope with the complexity of the system.

We developed an application using the tool for modeling and simulation JDEVS to simulate the user's preferences. As a future work, it would be very interesting to design a controller in order to update the fuzzy rules. It could be carried out by a feedback control strategy.

\section{REFERENCES}

[1] J. A. Orosa, "Research on general thermal comfort models," European Journal of Scientific Research, vol.29, no. 2, pp 217-227, 2009.

[2] F. Calvino, M. Gennusa, G. Rizzo, and G. Scaccianoce, "The control of indoor thermal comfort conditions: Introducing a fuzzy adaptive controller," Energy and Buildings, vol. 36, no. 2, pp. 97-102, February 2004.

[3] K. Chen, Y. Jiao, and E. Lee, "Fuzzy adaptive networks in thermal comfort," Applied Mathematics Letters, vol. 19, no. 5, pp. 420-426, May 2006.

[4] J. Liang and R. Du, "Thermal comfort control based on neural network for HVAC application," in Proc. 2005 IEEE Conf., 2005, pp. 819-824.

[5] A. Guillemin and N. Morel, "An innovative lighting controller integrated in a self adaptive building control system," Energy and Buildings, vol. 33, no. 5, pp 477-487, 2001
[6] V. Vainio and A. Vanhala, "Continuous-time fuzzy control and learning methods," in Proc. 7th International Symposium on Communications and Information Technologies, Sydney, Australia, 2007, pp. $346-351$.

[7] B. P. Zeigler. "Theory of modeling and simulation," Academic Press, 1976.

[8] A. G. Wainer and P. Mosterman, "Discrete-event modeling and simulation: Theory and applications," CRC Press, 2010, pp. 4-7.

[9] L. X. Wang, "A course in fuzzy systems and control," Prentice Hall PTR, 1997.

[10] L. A. Zadeh, "Fuzzy sets," Information and Control, vol. 8, no. 3, pp. 338-353, June 1965.

[11] The MultiCom research group. [Online]. Available: http://multicom.imag.fr/

[12] M. Gallissot, J. Caelen, N. Bonnefond, B. Meillon, and S. Pons, "Using the multicom domus dataset," LIG, Grenoble, France, Research Report RR-LIG-020, 2011.

[13] M. Gallissot, "Modéliser le concept de confort au sein de l'habitat intelligent: Du multisensoriel au comportement," PhD Thesis, University of Grenoble, France, 2012.

[14] J. B. Filippi and P. Bisgambiglia, "JDEVS: an implementation of a DEVS based formal framework for environmental modelling," August 2003.

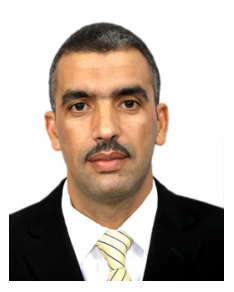

Lahcene Aid is a postgraduate student and researcher in the Department of computer science at University of science and technology of Oran-Algeria. His research interests include pervasive measurement systems for intelligent environments and adaptive, Intelligent Systems Modelling and Simulation. Graduated from the same University where he received his engineer diploma in 1999. He received his MSC (Magister) in computer information systems from Ibn Khaldun's University of Tiaret in 2011.

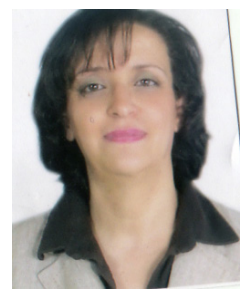

Lynda Zaoui is currently Associate Professor at the University of Science and technology of Oran-Algeria. She is responsible of Multimedia Object team in System-Signal and Data laboratory (LSSD). Her main researches are centered on manipulation of multimedia objects (multimedia mining, synchronization of multimedia documents, Content Based Image retrieval, information retrieval). Graduated from ES SENIA University where she received her engineer diploma in 1984. Thereafter, she gained an MSC (DEA) in modeling simulation from Paul Sabatier University (Toulouse) in 1985 and received Phd degree in computer Sciences in 1987 from the same University. She received a Doctorat degree in 2006 from the university of Science and Tchnology of Oran

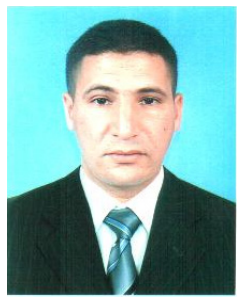

Sid Ahmed Mostefaoui was born in Algeria he is a postgraduate student and researcher in the Department of computer science at University of science and technology Oran-Algeria. His main research interests include software systems and adaptive control systems in smart spaces. Graduated from the same University where he received his engineer diploma in 2002. He received his MSC in computer information systems from Ibn Khaldun's University of Tiaret in 2010 